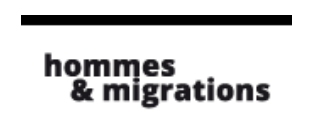

Hommes \& migrations

Revue française de référence sur les dynamiques

migratoires

$1284 \mid 2010$

Migrations et environnement

\title{
Le rôle des cadres normatifs et des organisations internationales
}

Une conversation avec Jean-François Durieux (HCR) et Philippe Boncour (OIM)

François Gemenne, Béatrice Cointe, Jean-François Durieux et Philippe Boncour

\section{OpenEdition}

Journals

\section{Édition électronique}

URL : http://journals.openedition.org/hommesmigrations/1255

DOI : 10.4000/hommesmigrations.1255

ISSN : 2262-3353

Éditeur

Musée national de l'histoire de l'immigration

Édition imprimée

Date de publication : 1 mars 2010

Pagination : 128-143

ISSN : 1142-852X

\section{Référence électronique}

François Gemenne, Béatrice Cointe, Jean-François Durieux et Philippe Boncour, «Le rôle des cadres normatifs et des organisations internationales », Hommes \& migrations [En ligne], 1284 | 2010, mis en ligne le 29 mai 2013, consulté le 01 mai 2019. URL : http://journals.openedition.org/

hommesmigrations/1255; DOI : 10.4000/hommesmigrations.1255 


\section{Le rôle des cadres normatifs et des organisations internationales}

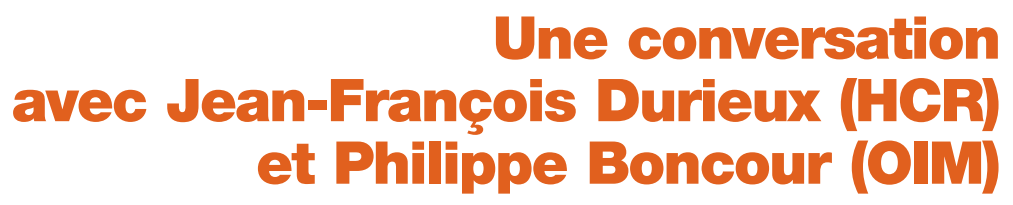

Entretien conduit par François Gemenne et retranscrit par Béatrice Cointe

Le Haut-Commissariat des Nations unies pour les réfugiés (HCR) et l'Organisation internationale pour les migrations (OIM)

sont en première ligne pour porter secours et assistance aux personnes déplacées par des catastrophes naturelles. Comment ces opérations trouvent-elles leur place dans le mandat de ces organisations ? Quels sont leurs rôles respectifs dans la gestion des crises et les instruments à leur disposition ? Jean-François Durieux est directeur de la Division de l'appui et de la gestion des programmes au sein du HCR, tandis que Philippe Boncour est directeur du Dialogue international sur les migrations au sein de l'OIM. Ils coprésident le groupe de travail spécial sur les migrations et déplacements de populations créé au sein de la task force "Changement climatique" du Groupe permanent inter-agences (IASC) ${ }^{(1)}$.

La rencontre de ces deux spécialistes de la question des migrations environnementales permet de faire le point sur l'organisation de la gestion de ces déplacements de population au plan international. 
François Gemenne : On a l'impression que le HCR a d'abord été un peu plus réticent que l'OIM à traiter la question du lien entre migration et environnement, mais l'a maintenant saisie à bras-le-corps. Comment expliquez-vous cette évolution ?

Jean-François Durieux : Il y a une douzaine d'années, il y avait des discussions à l'intérieur du HCR sur les questions de déplacés environnementaux. Ensuite, il y a eu une espèce de creux. L'attention s'est déplacée vers un débat plus global sur la question du déplacement interne, de ses rapports avec la protection des personnes qui traversent les frontières et le rôle du HCR dans cette problématique. Au sein des agences humanitaires des Nations unies, et même dans les agences de développement (à l'exception du Pnue ${ }^{(2)}$ et quelques agences spécialisées en matière d'environnement), la prise de conscience a été relativement lente sur le besoin d'intégrer le changement climatique dans nos politiques. Cela apparaît clairement en matière de migration "forcée" : c'est le débat sur le changement climatique qui a remis la question des déplacements causés par l'environnement à l'ordre du jour. On constate pour le moment une véritable déferlante à ce sujet.

\section{F. G. : Du côté de l'OlM, d'où est venu l'intérêt pour les relations entre migration et environnement ? L'OIM s'y est intéressée avant le HCR - pour quelles raisons ?}

Philippe Boncour : Je dirais plutôt "en même temps", parce que nos premières expériences dans ce domaine remontent respectivement à 1992 et à 1996, à l'occasion de deux conférences internationales à la fois dédiées au dialogue politique et à la recherche; et pour l'une des deux au moins nous avons travaillé directement avec le HCR - si ma mémoire ne me trahit pas - et pour l'autre avec le Refugee Group. Après ces deux conférences, nous avons commencé à mettre en place des programmes opérationnels. Le plus important d'entre eux, c'est évidemment l'opération humanitaire qui a suivi l'ouragan Mitch, en 1998 en Amérique centrale. Ce fut pour nous une très grosse intervention. Par la suite, nous avons essayé de théoriser la pratique en quelque sorte, c'est-à-dire tâcher de penser ensemble dialogue politique, recherche et action opérationnelle.

\section{F. G. : Jean-François Durieux, est-il également possible d'identifier pour le HCR quelques événements de ce type, qui ont marqué le début de l'implication de votre organisation sur cette question ?}


J.-F. D. : C'est le tsunami de 2004, et ensuite le tremblement de terre au Pakistan en 2005, qui ont représenté les premières opérations d'envergure du HCR dans ce domaine. Ces catastrophes consécutives ont fait réfléchir le HCR sur son implication et sur le besoin d'avoir et de préciser des "rules of engagement", ces règles concernant notre implication dans ces situations.

Le tsunami correspond évidemment à un concours de circonstances assez exceptionnel pour le HCR. D'abord c'était la catastrophe du moment, tout le monde y était. Même si l'on peut également parler d'Aceh et d'autres endroits, c'est vraiment notre action au Sri Lanka qui a solidifié, incarné l'implication du HCR dans les situations de catastrophes naturelles. Le Sri Lanka était une des rares opérations où le HCR travaillait depuis des décennies sur des questions de déplacements purement internes, sans que cette opération ne provoque aucune crise de conscience ${ }^{(3)}$ - c'était l'opération phare de nos actions en faveur des déplacés internes, et personne ne la mettait en doute. Comme le tsunami a frappé cette zone, on était déjà dans un modèle d'intervention tout à fait accepté et acceptable auprès des personnes déplacées à l'intérieur de leur pays. La différence, bien sûr, c'est qu'avant elles étaient déplacées par le conflit, et qu'elles l'étaient désormais par les effets conjugués du conflit et du tsunami. Le HCR était là avec des moyens matériels et humains mobilisés nulle part ailleurs.

P. B. : L'OIM était la seule organisation internationale à être déjà présente à Aceh avant le tsunami, ce qui nous a coûté cher, mais aussi permis d'être une sorte de tête de pont, du moins dans les premiers temps. Nous étions présents également au Myanmar par le biais de programmes touchant à la santé et au bien-être des travailleurs dans cette zone, et bien sûr aussi à Haïti. Dans chaque cas, nous nous sommes déployés extrêmement rapidement. En Haïti, on a dû passer en moins de deux semaines de 67 personnes sur le terrain à plus de 215. C'est très important de le signaler, pour nos deux organisations : certes, nous réfléchissons et menons une recherche, mais la priorité reste d'apporter assistance et protection aux personnes qui en ont besoin. Par ailleurs, tous les travaux de recherche disponibles aujourd'hui montrent que c'est bien du côté des déplacements internes de populations que s'effectue l'essentiel des mouvements liés aux conséquences du changement climatique ou de la dégradation de l'environnement. Rien ne porte à croire que cette réalité va évoluer fondamentalement dans les années qui viennent, malgré un certain alarmisme politique dans le cadre de débats nationaux parfois très tendus. Pour terminer sur ce point, s'agissant des personnes déplacées de l'intérieur, nous bénéficions aujourd'hui, en 2010, d'un instrument qui date de 1998 : les Principes directeurs sur les déplacements internes. 
Comme sur beaucoup d'autres terrains, nous avons avec le HCR une division des responsabilités qui est assez claire globalement, même si, bien sûr, localement il y a des ajustements qui se mettent en place. Grosso modo, lorsque ces déplacements internes sont liés à des conflits, c'est plutôt le HCR qui assume le leadership de l'opération - l'OIM y participe également, bien sûr, avec d'autres organisations - et lorsqu'il s'agit de déplacements internes liés aux catastrophes naturelles ou plus généralement à la dégradation de l'environnement, c'est l'OIM qui assure ce leadership, ce qui n'est nullement exclusif. Ce partage des responsabilités fonctionne finalement assez bien.

\section{F. G. : On a l'impression qu'il y a eu au sein du HCR une sorte de "crise de conscience" quant à l'implication de l'organisation dans les migra- tions environnementales. Est-ce une question liée au mandat du HCR, ou était-ce davantage une question de personnel, de budget, ou de culture de l'organisation simplement?}

J.-F. D.: C'est au départ essentiellement une question de mandat. Le HCR s'intéresse spécifiquement, pour de bonnes raisons, aux personnes qui se trouvent en dehors de leur pays. Le problème international hier comme aujourd'hui, c'est d'abord le problème des réfugiés. Bien sûr, lorsqu'on parle de conflits, ou d'autres causes de déplacement, la plupart des situations comportent à la fois un mouvement de déplacement interne et externe. Il se produit une espèce de spill-over: les gens commencent à se déplacer à l'intérieur des territoires et certains arrivent à franchir une frontière pour trouver une meilleure sécurité.

Pour que le problème des déplacements internes soit traité au niveau international, il a fallu un long, très long détour par la question de la violation des droits de l'homme. La communauté internationale ne pouvait pas rester insensible à ce qui se passait à l'intérieur des pays - y compris le déplacement, mais qui était en fait un symptôme des problèmes, et non leur cause. À mon avis, on n'a jamais dépassé ce dilemme de savoir si le déplacement accroît les vulnérabilités ou au contraire les réduit puisque cela varie au cas par cas. Dans certains cas, les gens se déplacent en cherchant une meilleure protection qu'ils vont peut-être trouver à l'intérieur même de leur propre pays. Dans d'autres cas, en se déplaçant, ils accroissent encore leur vulnérabilité.

P. B. : Cette question des droits de l'homme me parait absolument centrale lorsqu'on parle de ce type de migrations, tout simplement parce qu'en l'absence d'un régime de protection internationalement reconnu pour les personnes qui sont amenées ou 
contraintes à sortir des frontières de leur État pour des raisons majoritairement ou uniquement liées au changement climatique ou à l'environnement, il n'existe pas d'instrument contraignant spécifique. Par conséquent, sur quoi pouvons-nous, les uns et les autres, nous appuyer? Sur les instruments qui sont liés aux droits de l'homme. Il peut y avoir dans certains cas des formes d'accords bilatéraux, voire régionaux, mais en général ces accords n'ont pas été initialement prévus ou conçus pour s'appliquer spécifiquement à cette population. Nous mesurons vraiment les limites que pose sur le terrain l'absence d'un cadre plus spécifique.

\section{F. G. : L’action de l'OIM sur ce terrain a-t-elle également été mar- quée par des crises de conscience, des débats sur l'opportunité d’intervenir?}

P. B. : Crises de conscience, non. Débats, oui, parce que lorsque nous avons été amenés pour la première fois à aborder cette question dans le cadre de nos organes de gouvernance au Conseil de l'OIM de novembre 2007. Certains de nos États membres se sont en effet demandés pourquoi cette question arrivait sur l'agenda. Nous avons dû expliquer que nous ne traitions pas du changement climatique et de la dégradation de l'environnement en tant que tels, mais de leur impact sur les mouvements de population à l'intérieur d'un pays, dans une région donnée ou au niveau transcontinental. Et cette légitimité a été d'autant plus facilement acquise que nous pouvions nous appuyer sur la science, et en l'occurrence sur deux rapports d'évaluation du GIEC, le premier de 1990 et le quatrième de 2007, où à deux reprises il était clairement établi que l'un des impacts les plus importants du changement climatique concernerait en effet les mouvements de population.

\section{F. G. : Quel rôle a chacune de vos deux organisations aujourd'hui sur le terrain ? S'agit-il essentiellement d'une réponse humanitaire à des situations d'urgence, ou développez-vous également des program- mes d'accompagnement des migrations liées à des dégradations plus lentes de l'environnement, à des questions de réinstallation, etc. ?}

J.-F. D. : Nous avons effectivement des programmes de gestion des ressources, pour déplacer des camps de réfugiés suite à des inondations, et nous faisons face, dans de nombreux pays, et notamment en Afrique, à des phénomènes de désertification, de dégradation de l'environnement, de perte des ressources en eau... On gère ces problèmes avec les communautés locales, mais essentiellement sur la base de notre raison d'être, c'est-à-dire parce qu'il y a là-bas des personnes qui sont sous notre 
protection. Nous ne sommes pas encore passés au niveau où l'on transposerait ce savoir-faire, qui existe, à des populations plus larges.

Par contre, en matière d'aide d'urgence, c'est vrai que le HCR a été de plus en plus impliqué, à défaut de l'être systématiquement, dans des réponses interagences - mais on parle ici essentiellement de déplacés internes. Car le HCR a un rôle à jouer en matière de protection, d'abri, de gestion des camps, avec des partenaires différents. La mécanique qui a permis ce développement est la fameuse approche modulaire. Dans le cas d'Haïti par exemple, le HCR a prêté main-forte à l'OIM au début ; nous avons également déployé des collègues spécialisés en protection vers le Haut-Commissariat des droits de l'homme, qui coordonne le module de protection. Le HCR a opté pour une politique de visibilité très réduite, mais a accepté de jouer le jeu interagences et de prêter main-forte là où c'était nécessaire. Et en même temps - ce qui à mon avis explique bien la place où le HCR pense pouvoir se situer dans toutes ces problématiques - il a aidé à créer et coordonne aujourd'hui un module de protection en République dominicaine. Effectivement, le contexte haïtien implique un aspect transfrontalier : les gens traversent la frontière pour aller se ravitailler ou se faire soigner. On se trouve là sur un terrain mieux connu pour le HCR : celui de la gestion des relations entre États et des phénomènes de migration, même circulaires, mais qui touchent plusieurs États.

\section{F. G. : Philippe Boncour, comment identifieriez-vous les spécificités de l'OIM en matière opérationnelle ?}

P. B. : La situation est sensiblement différente pour l'OIM, et d'une façon assez naturelle, précisément pour les raisons de mandats respectifs que Jean-François Durieux a abordées auparavant. Chez nous, un premier élément important de réflexion est de constater que sur les trente dernières années, 1,6 milliard de personnes ont été déplacées par la sécheresse seule, alors que 718 millions - j’allais dire 718 millions "seulement" - ont été déplacés par des catastrophes naturelles. Ces déplacements de personnes quantitativement plus importants dans le cadre de processus graduels plutôt que suite à des événements catastrophiques vont se poursuivre. Il faut en tirer les enseignements.

En préparant Copenhague, avec le HCR, l'OCHA ${ }^{(4)}$, l'Institut pour l'environnement et la sécurité humaine de l'Université des Nations unies, le Conseil norvégien des réfugiés et d'autres, nous nous sommes dit qu'il serait important de regarder cette ligne d'horizon. Il nous fallait prendre en compte tout ce que nous avions fait de manière un peu éparse, un peu partout dans le monde, en termes de projets, justement pour pouvoir disposer d'indicateurs, mieux lire notre propre action, et l'inscrire dans 
la durée et sur un mode plus prévisionnel. C'est ce qui a donné jour au compendium de nos activités liées au changement climatique. Ce compendium est classé par régions, par types d'événements ou de processus, par types de réponses également. En compilant les données, nous nous sommes aperçus que sur la dernière décennie, nous avions mené pas moins de 500 projets autour de ces questions. Et contrairement à la perception qu'on pouvait en avoir a priori, si 54 \% de ces projets étaient certes liés à l'aide humanitaire d'urgence, 46 \%, déjà aujourd'hui, étaient liés à l'adaptation au changement climatique, que ce soit par la stabilisation des communautés, les programmes de migrations temporaires ou circulaires avec éligibilité ciblée sur ce type de populations, ou d'autres moyens encore orientés vers le développement durable. Nous essayons en effet de couvrir l'ensemble du continuum qui va de la réponse d'urgence en cas de catastrophe jusqu'à l'adaptation au changement climatique sur place, lorsque c'est possible. Quand ce n'est pas possible, nous envisageons la planification technique de processus de réinstallation qui pourraient être éventuellement mis en ceuvre si des États souverains, d'un commun accord, les approuvaient.

J.-F. D. : Je suis prudent. Le HCR, tout comme l'OIM, sait faire de la réinstallation, techniquement parlant. Mais on sait le faire dans des contextes qui aujourd'hui sont clairement définis. Demain, ce ne sera pas la technicité qui nous fera éventuellement défaut, mais plutôt la détermination d'un État à accepter ou à demander l'aide d'une organisation internationale, d'un partenaire extérieur, pour reloger ou réinstaller des déplacés sur son propre territoire. En aucun cas la décision politique de la réinstallation ne peut nous appartenir, même s'il est important que nous soyons préparés au plan technique à y répondre. Cela soulève un point vraiment déterminant pour le HCR : il s'agit du rôle des États sur ces questions migratoires, et notamment le rôle de leur souveraineté.

Si nous récusons le terme de "réfugié climatique", ce n'est pas seulement parce que nous ne l'aimons pas, c'est surtout parce que les États d'origine eux-mêmes ne veulent pas être vus comme des États qui produisent des réfugiés, et cela me paraît tout à fait sain. On n'est pas ici dans un scénario où la communauté internationale, par le biais d'agences ou d'autres modalités d'intervention, serait vue comme confisquant une partie de la souveraineté des États, en disant: "Vous n'êtes pas capables de gérer vos populations, donc on va s'en occuper pour vous." Et il ne faut pas que nous en arrivions à ce scénario. On n'est pas du tout dans la même configuration ici, surtout dans les phénomènes lents.

Dans les situations de catastrophes, comme on l'a vu en Haïti, il est vrai que la gouvernance, qui était déjà faible, s'est complètement écroulée. Ceci dit, personne n'avait l'intention de se substituer au gouvernement haïtien : c'était plutôt qu'il y 
avait un vide à remplir, même si on peut certainement imaginer une situation où une catastrophe naturelle pourrait être l'occasion d'un changement de régime. Lorsque le cyclone Nargis a frappé le Myanmar, des États étaient prêts à aider et avaient également cette idée de substitution à leur agenda - ce n'était sûrement le cas ni de l'OIM ni du HCR, on est plus réalistes que ça. Mais, en tout cas, lorsqu'on travaille sur des phénomènes dans la durée - par exemple la sécheresse, la désertification, ou la perte de territoire - il faut essayer de maintenir ces États dans une situation où ils peuvent, de façon souveraine et responsable, exercer leur autorité et leur rôle dans des négociations bilatérales ou multilatérales. Mais on est là dans un rôle assez différent d'une situation de réfugiés où il est clair que l'État d'origine est, en tout cas pour un temps, mis à l'écart.

P. B. : S'il n'y a pas encore d'instrument mondial spécifique qui permettrait de mettre tout le monde d'accord, un certain nombre de pays ont quand même réfléchi à l'impact potentiel des catastrophes naturelles, voire de processus plus lents. Ils ont mis en place des systèmes qui, pour être temporaires, n'en offrent pas moins une protection aux personnes déplacées par ces événements. Les États-Unis ont commencé avec la mise en place d'un statut de protection temporaire, précisément à la suite de l'ouragan Mitch que j'ai évoqué tout à l'heure. Ce statut a permis à un certain nombre de ressortissants d'Amérique centrale et d'Amérique latine d'obtenir un statut légal aux États-Unis; beaucoup d'entre eux l'ont conservé jusqu'à aujourd'hui. Suite à la catastrophe en Haïti, on a encore eu recours à cet instrument. D'autres pays, notamment européens, dans une réflexion un peu similaire, ont inscrit dans leur dispositif législatif des régimes qui permettent d'accorder un statut de protection temporaire, parfois avec des droits plus étendus. C'est une piste qui me semble assez prometteuse parce qu'elle ressort de la démarche souveraine et volontaire d'un État, qui décide de prendre en compte cette migration particulière qui n'est pas générée par une persécution. Certes, la nature peut être cruelle, mais à ma connaissance, elle n'a jamais frappé intentionnellement.

\section{F. G. : Quelle est l'attitude des gouvernements vis-à-vis de l'action de vos organisations sur ces théâtres de migrations environnementales : ont-ils plutôt tendance à vous demander d'intervenir davantage, ou de rester en dehors de ces questions ? Y a-t-il éventuellement une différence entre le Nord et le Sud?}

J.F. D. : Oui, il y a une différence entre le Nord et le Sud si on considère le Nord comme étant le monde riche, et donc nos donateurs. Il n'y a pas une frilosité, mais 
quelques hésitations, qui ne sont pas nouvelles. Une réalité avec laquelle le HCR doit vivre constamment. La perception d'un risque d'élargissement du mandat soulève toutes sortes de questions, et notamment la crainte, bien sûr, de voir le HCR faire des appels de fonds beaucoup plus importants. Ce genre de dialogue est toujours très difficile. C'est une fausse question, mais il est très difficile de la présenter comme telle à nos donateurs. Soit il y a des ressources pour régler ce genre de situations, soit il n'y en a pas. S'il y en a, il faut que les donateurs, le système des Nations unies, tous les systèmes de coordination qui existent, trouvent les meilleurs instruments, les meilleures synergies. Il faut rester dans une logique d'efficacité, bien sûr, et de moindre coût. L'augmentation du financement du HCR n'est pas à mon avis une question indépendante de celle du financement humanitaire, ni de la gestion des migrations en général. Il s'agit de savoir comment on va répartir ensuite cette manne, si manne il y a. Mais la frilosité de la part des donateurs demeure.

Pour ce qui concerne les pays du Sud, notamment ceux affectés par le changement climatique causant des déplacements de populations difficiles à gérer, je dirais que l'attitude est généralement assez ambivalente en ce qui concerne le HCR, et cela ne m'étonne pas. D'un côté, il y a effectivement cette crainte de mettre une étiquette "réfugiés" sur des phénomènes qui ne le sont pas, avec toute la charge politique et négative que cela comporte, et donc le HCR n'est peut-être pas vu comme l'interlocuteur le plus évident. D'un autre côté, comme avec les Maldives, le Bangladesh et d'autres, ces pays apprécient l'écoute dont bénéficie le HCR auprès de la communauté internationale. Même si un État comme le Bangladesh a, pour toutes les raisons que j'ai indiquées, des réticences à brandir trop haut la bannière des "réfugiés climatiques", ces pays se rendent compte qu'ils peuvent, à travers le HCR, attirer l'attention sur un problème qui est réel.

\section{F. G. : Et du côté de l'OIM ? Ce souci autour du terme "réfugié" est sans doute moins présent...}

P. B. : Par définition. Mais en même temps, nous savons que les États du Nord ou du Sud entendent bien conserver la main sur les débats à caractère politique et les mesures concrètes qui doivent éventuellement être prises. J'en veux pour preuve l'adoption par l'Assemblée générale des Nations unies, en juin 2009, après un très long débat, d'une Résolution sur le changement climatique et ses possibles implications en matière de sécurité. Cette résolution demandait au Secrétaire général de produire un rapport sur cette question, ce qu'il a fait. L'OIM, tout comme le HCR, ont contribué à ce rapport, dans leurs domaines de compétence. Le Secrétaire général a identifié dans le rapport cinq risques majeurs. Parmi ces risques, deux sont directement liés aux questions migratoires ou au mandat du HCR : le premier rappelle que la pression 
sur les ressources en eau ou les terres agricoles peut générer des conflits entre communautés, notamment entre nomades pastoralistes et agriculteurs; le second pointe les nouvelles conséquences de la disparition éventuelle d'États-nations sur l'apatridie, jusqu'à conduire à une nouvelle définition de celle-ci.

Au Conseil de sécurité, le débat n'est pas fini. Certains estiment qu'il faut en effet regarder le changement climatique et son impact sur les déplacements de personnes comme un risque pour la sécurité des États, d'autres penchent plutôt en faveur de l'idée que le risque majeur pèse avant tout sur la sécurité humaine, et non sur celle des États. Or, ces deux aspects sont l'un et l'autre importants. Mais certains se demandent si le Conseil de sécurité est le lieu de discussion idoine. La question reste posée. Ce n'est évidemment pas à l'OIM ni au HCR d'y répondre, mais il existe aussi des tenants d'une approche par le biais de l'Ecosoc, le Conseil économique et social des Nations unies, où justement ces questions de sécurité humaine pourraient être remises au centre du débat.

Il y a des instances qui sont plus claires dans leur position. Pour l'Union européenne par exemple, deux étapes récentes sont importantes. La première, c'est le rapport dit "rapport Solana" de 2008, qui était un rapport conjoint de la Commission européenne et du Haut Représentant pour la politique étrangère et de sécurité, où l'on aborde très ouvertement et directement la question des migrations climatiques comme une menace de sécurité pour l'Union européenne. Le papier blanc plus récent, de juillet 2009 porte également sur cette question: "Adaptation au changement climatique : vers une approche européenne".

Clairement, c'est une affaire de discussions entre États. Or, parfois, les intérêts des pays développés et des pays en développement divergent, et à d'autres moments au contraire, et sur le même sujet, ils peuvent converger. Un point de convergence possible, évoqué brièvement tout à l'heure, est de penser qu'on peut diminuer la pression sur les écosystèmes et les populations dans les zones affectées par une dégradation environnementale en permettant à un groupe donné de personnes de se rendre dans un autre pays - éventuellement d'ailleurs un pays de l'Union. Là-bas, ces personnes pourraient travailler dans le secteur agricole, dans celui des services à la personne, de l'hôtellerie et de la restauration, du BTP... et envoyer des transferts de fonds vers leur famille. Donc si on s'y prend à temps, à un stade encore peu avancé de dégradation de l'environnement, les migrations peuvent être une réponse.

Évidemment, plus la question est aiguë, plus l'attention internationale est focalisée, plus les fonds parviennent facilement. Mais il n'y a pas de récurrence budgétaire. Et plus on va vers le soutien à des processus de développement durable, forcément générateurs de récurrence budgétaire, plus il est difficile d'attirer des financements. Le politique est comptable de l'utilisation des deniers nationaux devant son opinion 
publique, à laquelle il n'est pas forcément facile d'expliquer, dans les contextes économiques tendus que l'on connaît, qu'une solidarité internationale doit se manifester ailleurs nécessairement.

J.-F. D.: Il est vraiment très difficile aujourd'hui d'arriver à motiver les États sur le long terme. Le changement climatique agit comme une sorte de sonnette d'alarme dont on a besoin de temps en temps pour relancer ce qui devrait être des réflexes de solidarité au sens à la fois le plus noble, mais aussi le plus utilitaire du terme. Au final, le développement nous intéresse tous : il s'agit de sauver notre planète, bien entendu, mais aussi de trouver des équilibres, d'essayer d'établir un petit peu de justice dans ce monde... Le débat sur le développement est devenu d'une tristesse absolue. En tant qu'humanitaires, on est de plus en plus confrontés aux conséquences de cet appauvrissement : de très nombreuses questions relèvent du développement, mais nous sont présentées comme des questions humanitaires, y compris celle des migrations environnementales. Pourquoi ? Parce qu'on attend que la situation dégénère au point où effectivement, la seule solution possible devient l'humanitaire. Mais comme le disait très bien Cornelio Sommaruga, je crois, il n'existe pas de catastrophe humanitaire. Les conséquences sont effectivement terribles pour l'humanité, mais la catastrophe a toujours d'autres causes qui sont souvent des causes politiques. On a permis que la situation dégénère à un point où l'on en est réduit à sauver des vies in extremis, et à ce moment-là c'est dix fois plus difficile de reconstruire dans la durée.

P. B. : Nous avons évoqué l'appauvrissement dans son acception humaine. À la conférence sur le financement du développement à Monterey en 2002, puis à celle de Doha en 2008, on a réitéré des engagements politiques visant notamment à ce que les pays développés consacrent 0,7 \% de leur PIB à l'aide publique au développement. Seuls cinq pays dans le monde ont à ce jour honoré leurs engagements. J'aborde ce point car il garantit un financement dans la durée.

Or,à Copenhague, récemment, des engagements ont été pris à hauteur de 30 milliards de dollars en faveur de l'adaptation au changement climatique dans les pays en développement pour les trois prochaines années. Le Programme des Nations unies pour le développement a estimé le coût annuel de l'adaptation au changement climatique à 86 milliards de dollars. Donc il apparait déjà un problème structurel de sous-financement. Mais quel financement est disponible aujourd'hui, objectivement ? À ma connaissance, il n'y a guère que ce qui se trouve dans l'enveloppe du Fonds mondial pour l'environnement, c'est-à-dire un total cumulé inférieur à 400 millions de dollars. Donc, les engagements sont évidemment nécessaires, mais il faut les tenir, ce qui est mieux. 
J.F. D. : Évidemment, les engagements se trouvent très fortement dévalués si on ne les respecte jamais.

\section{F. G. : Quelle place peut avoir l'environnement dans le débat sur la gouvernance internationale des migrations?}

J.-F. D.: On observe, ici comme ailleurs, un point de convergence entre deux enjeux globaux. D'un côté, il y a effectivement toute cette discussion sur le mécanisme de gouvernance des migrations ; de l'autre côté, il y a toute une discussion au moins aussi épineuse sur la question de la gouvernance de l'environnement. Mais au croisement de ces enjeux, se trouvent, d'une part, les phénomènes migratoires, dont certains ont effectivement une relation avec l'environnement, et, d'autre part, la question de la gestion des phénomènes environnementaux, maintenant climatiques. Dans ce domaine, on compte la Convention-cadre sur le changement climatique, mais aussi toutes les autres conventions, le Global Environnement Facility $y^{(5)}$ qui a ses propres problèmes... Donc, on est en train de jongler avec des balles...

P. B. : C'est à la croisée des chemins entre deux notions qui elles-mêmes sont à la croisée des chemins entre beaucoup d'autres notions... Cela explique pourquoi la communauté internationale a beaucoup de difficultés avec cela.

\section{F. G. : Il y a en ce moment, dans les milieux académiques, un foison- nement de propositions pour le développement de nouvelles conven- tions, de nouveaux instruments politiques, de nouvelles agences... Est-il nécessaire de développer de nouveaux instruments? Comment voyez-vous à l'avenir le rôle et l'activité de vos organisations dans ce débat ?}

J.-F. D. : En ce qui concerne le HCR, nous identifions deux chantiers sur lesquels nous sommes prêts à travailler. Le premier n'est pas nouveau, mais a pris une dimension nouvelle dans le contexte du débat sur le changement climatique : il s'agit de la coordination interagences, notamment sur la protection des victimes de catastrophes naturelles. Beaucoup de travail peut être fait en amont des catastrophes, pour s'assurer que les États, qui se trouvent en première ligne et sont pleinement responsables et souverains, ont les procédures, les mécanismes, et le savoir-faire nécessaires pour faire face à des questions telles que la séparation des familles, la perte de documents d'identité, de registres civils, ou même des vulnérabilités, des discriminations qui peuvent ressurgir, etc. 
Il y a également du travail à effectuer sur les éléments de réponse et d'appui à donner à ces questions de protection. Si les trois agences qui partagent cette responsabilité le Haut-Commissariat aux droits de l'Homme, l'Unicef et le HCR - ne parviennent pas à s'entendre quant à leurs rôles respectifs et aux mécanismes de coordination, le HCR serait prêt à monter au créneau, peut-être plus rapidement que les autres. Cela reste à prouver en pratique, mais nous nous sommes engagés à travailler dans cette voie-là. Sur le plan conceptuel et sur le plan normatif, lorsqu'on parle de protection des déplacés internes, on a déjà les Principes directeurs sur les déplacements internes, ainsi qu'une certaine expérience dans le domaine.

Le deuxième grand chantier concerne les personnes déplacées qui, à un moment donné, ne peuvent pas rentrer chez elles, à cause de la perte de leur habitat. Il peut s'agir de personnes qui se trouvent dans un pays étranger lorsque la catastrophe frappe, mais aussi de personnes qui sont déplacées hors de leur pays par des phénomènes de plus longue durée, c'est-à-dire des années ou des décennies. Ce phénomène est difficile à quantifier, notamment en raison précisément de la durée que peuvent prendre ces déplacements, mais il est vrai qu'il existe une lacune juridique, qui est en partie comblée par le genre de mécanismes auxquels nous faisions allusion tout à l'heure, comme le régime de protection temporaire aux ÉtatsUnis. Pour le moment, ces mécanismes s'adressent essentiellement à la question du non-retour. Or, ce n'est pas la même chose d'admettre les gens ou de retarder provisoirement leur déportation ou leur expulsion. Le HCR a accepté de se saisir de cette question, évidemment en accord avec ses partenaires. Dans le cadre du $60^{\mathrm{e}}$ anniversaire du HCR et de la Convention sur les réfugiés et également du $50^{\mathrm{e}}$ anniversaire d'une des deux conventions sur l'apatridie, le HCR a décidé d'inscrire ces questions à l'agenda d'un dialogue intergouvernemental que nous allons organiser cette année et l'année prochaine.

P. B. : Contrairement au HCR, l'OIM ne dispose pas d'un mandat à exécuter et n'a pas davantage de rôle normatif. Notre mandat consiste à gérer les migrations le mieux possible, de façon ordonnée, humaine et sûre. C'est ce qui figure dans notre constitution.

Évidemment, la communauté universitaire - en France, en Europe, ailleurs, dans le Pacifique notamment - réfléchit, travaille sur des projets. Certains sont clairement formulés comme des projets de conventions, d'autres comme des projets de protocoles additionnels, d'autres encore comme des projets d'instruments de soft law. Pour ma part, je pense que cette réflexion est stimulante, et que c'est bien le rôle de la communauté universitaire, comme celui de la société civile, de s'y investir. Mais, par ailleurs, chacun doit rester dans son rôle. Jean-François Durieux disait tout 
à l'heure qu'il y aurait clairement - et il y a déjà - des réticences très fortes des États quant à l'expansion du champ d'application de la Convention de Genève de 1951. Je pense qu'il y aurait sans doute davantage de réticences encore à l'éventuelle mise en place d'une nouvelle convention qui prendrait en compte de façon spécifique ces populations. Au final, nous l'avons dit et répété l'un et l'autre, toute décision d'avancer dans ce domaine relève de la souveraineté des États. Il s'agit donc d'agiter des idées, de faire émerger les hypothèses possibles. C'est le sens de certaines initiatives prises récemment: je pense notamment à l'organisation de ce débat auquel nous participions l'un et l'autre, à l'initiative du Pr. Kälin, le représentant spécial du Secrétaire général de l'Onu pour les droits des déplacés internes, débat auquel avait aussi été invité le Pr. Marguénaud, qui venait précisément présenter aux États un projet de convention que son université(b) avait préparé.

J.F. D. : Il y a effectivement des lacunes juridiques. Elles ne sont peut-être pas celles que tous les universitaires identifient et peut-être pas aussi vastes, mais elles existent. Mais, stratégiquement, il ne suffit pas d'identifier les lacunes pour arriver nécessairement à identifier la forme de codification qui a des chances de fonctionner. En ce sens, la référence aux Principes directeurs est très bonne, parce que c'est un effort qui a été mené à bien, avec des investissements majeurs. Mais comme on l'a bien vu à cette occasion, lorsque l'on parle de déplacements d'une façon générale, ou de mobilité ou de migrations, on entre dans un domaine où le droit est peu présent. Et il y a de bonnes raisons pour cela. Encore une fois, méfionsnous des analogies avec le droit des réfugiés. Il y a de très bonnes raisons historiques et juridiques pour lesquelles nous avons un régime étroit; nous sommes les premiers à le dire, c'est presque un régime de faveur pour certains migrants. Pourquoi ? Parce qu'il se trouve que ces migrants créent un problème particulier au niveau interétatique qu'il faut résoudre.

En comparant deux approches possibles, l'une qui préconiserait une nouvelle convention, et l'autre qui plaiderait pour un élargissement de la Convention de Genève de 1951, je préfère quand même la première, parce qu'essayer d'élargir la Convention de 1951 équivaudrait à aller droit dans le mur. Une nouvelle convention, même si le monde ne semble pas encore mûr, c'est une façon de montrer ce qui peut être codifié. Ensuite il faut laisser la question aux vrais experts, c'est-à-dire aux politiques : ils décideront comment on va faire avancer ce débat pour arriver à quelque chose, qui pourra être du soft law ${ }^{(7)}$ du hard law, ou une combinaison des deux. À un certain moment, le monde académique doit non pas s'incliner, mais accepter que la démarche entre dans le domaine politique et doit être gérée comme telle. 
P. B.: Jean-François Durieux a évoqué à plusieurs reprises, mais sans entrer dans le détail, le travail interagences. Si on veut situer nos actions respectives, il est sans doute important de signaler qu'il existe au sein du Groupe permanent interagences une task force spécifique qui traite des questions de changement climatique. Cette task force réunit à intervalles réguliers les 23 membres du Groupe permanent, et les chefs d'agence ont décidé de formaliser, au sein de cette task force, un groupe de travail sur les questions de migrations et de déplacement, groupe dont Jean-François Durieux et moi-même sommes les coprésidents. Bien que notre action soit différente, elle est clairement complémentaire, et constitue une forme de leadership sur ces questions qui est acceptée de la part des autres agences. J'ajouterai que là où les universitaires, la société civile et les ONGs ont leurs préoccupations, là où les États ont également les leurs, nous apportons une expertise technique qui offre l'avantage de combiner le dialogue politique, la recherche et l'action opérationnelle. Nous pouvons, quand on émet une opinion sur la question des besoins en matière de protection, procéder à ce que les Anglo-Saxons appellent le reality check sur le terrain.

\section{Notes}

1. Inter-Agency Standing Committee, structure de coordination des opérations humanitaires de plusieurs organisations internationales telles que le HCR, l'OIM, l'UNICEF, l'OMS, la Croix Rouge, etc.

2. Programme des Nations Unies pour l'Environnement.

3. Techniquement, il n'entre pas dans le mandat du HCR d'intervenir "automatiquement" dans des situations de déplacements internes. Avant que l' "approche modulaire" ait été déployée, une interprétation large de son mandat l'avait pourtant déjà amené à intervenir dans de telles situations, parfois au prix de nombreuses interrogations

et "crises de conscience".

4. Bureau des Nations Unies pour la Coordination des Affaires Humanitaires.

5. Organe financier de la Banque mondiale en matière d'environnement.

6. Luniversité de Limoges, voir l'article de Julien Bétaille dans ce numéro.

7. Contrairement au hard law, le soft law est un droit non contraignant. 